\title{
Optical fibers as beam shapers: from Gaussian beams to optical vortices
}

\author{
Rita S. Rodrigues Ribeiro, ${ }^{1,2, *}$ Pabitra Dahal, ${ }^{2}$ Ariel Guerreiro, ${ }^{1}$ Pedro Jorge, ${ }^{1}$ and Jaime Viegas ${ }^{2}$ \\ ${ }^{1}$ INESC TEC, Rua do Campo Alegre, 687, Porto, Portugal and Departamento de Física e Astronomia, Faculdade de Ciências, \\ Universidade do Porto, Rua Campo Alegre, 687, Porto, Portugal \\ ${ }^{2}$ Masdar Institute of Science and Technology, Abu Dhabi, United Arab Emirates \\ *Corresponding author: arsr@inescporto.pt
}

Received 15 March 2016; revised 3 April 2016; accepted 5 April 2016; posted 7 April 2016 (Doc. ID 261174); published 2 May 2016

\begin{abstract}
This Letter reports a new method for the generation of optical vortices using a micropatterned optical fiber tip. Here, a spiral phase plate $(2 \pi$ phase shift) is micromachined on the tip of an optical fiber using a focused ion beam. This is a high resolution method that allows milling the fibers with nanoscale resolution. The plate acts as a beam tailoring system, transforming the fundamental guided mode, specifically a Gaussian mode, into the Laguerre-Gaussian mode $\left(\mathbf{L G}_{\mathbf{0 1}}\right)$, which carries orbital angular momentum. The experimental results are supported by computational simulations based on the finite-difference time-domain method. ( 2016 Optical Society of America
\end{abstract}

OCIS codes: (060.2310) Fiber optics; (140.3300) Laser beam shaping; (050.4865) Optical vortices.

http://dx.doi.org/10.1364/OL.41.002137

The nature of the angular momentum of light was initially investigated by Poynting in 1909, when he proposed that beams with circular polarization possess angular momentum [1]. Evidences supporting this phenomenon were published in 1936, where a birefringent wave plate was rotated, due to the angular momentum of light, while transforming left- into right-hand circular polarization, and vice-versa [2]. Currently the angular momentum linked with the circular polarization is mentioned as the spin angular momentum (SAM). Later on, in the 1990s, Allen et al. revealed that optical beams with an azimuthal phase dependency $(\exp (i l \phi))$, i.e., helical beams, carry orbital angular momentum (OAM) [3]. Helical beams are represented by Laguerre-Gaussian modes $\left(L G_{p, l}\right)$, which are the solutions of the paraxial wave equation in cylindrical coordinates [4]. These beams are characterized by indices $p$ and $l$, where $p$ is the radial index and defines the number of radially distributed field amplitude nodes; $l$ is the azimuthal index, or topological charge, and expresses the number of $2 \pi$ phase shifts per helicoid pitch. These beams are generally mentioned in the literature as optical vortices $(\mathrm{OVs})$ due to the screw dislocation along the axial direction and their phase singularity at the center [5]. Such features make OVs of broad and current interest. To give some examples, beams carrying OAM have been employed in a diversity of applications, ranging from optical trapping and rotation of particles [6,7], to the manipulation of atoms [8]. Simultaneously, quantum communication or rheological and viscometric methods have also taken advantage of their unique features [9]. Typically, the techniques used to generate OVs are based on free-space bulk setups, using diffractive gratings [10] via spatial light modulators $[11,12]$ or specially designed optical components, such as cylindrical [13] or spiral phase plates (SPP) $[14,15]$.

More recently, optical fibers have been used in the generation and propagation of OVs foreseeing a large number of distinct applications such as polarization maintaining fibers, nano-imaging, and quantum entanglement, as extensively reviewed in reference [16]. For instance, specially modified fibers with helical cores have been theoretically studied and experimentally explored in the generation of optical singularities [17-19]. However, the use of optical fibers as alternatives to bulk setups still has room for improvement. Besides this, the implementation of these systems as optical fiber tweezers (OFT) is also an unexplored opportunity with a large potential. As can be seen over the last decades, the use of OFT has been increasing, and more sophisticated and specialized systems have been developed [20]. For example, axicon-shaped optical fibers have been used since the advent of optical trapping using optical fibers. These structures can be easily fabricated using methods such as chemical etching and focused ion beam milling [21,22]. Axicon structures are able to transform Gaussian beams into Bessel beams, which are nondiffracting beams allowing, for instance, the simultaneous trapping of single and multiple particles. Alternatively, using optical fibers to generate $\mathrm{OV}$ is suitable for more complex manipulation of the target, such as rotation, without further assistance due to the transfer of orbital angular momentum. Therefore, these approaches complement each other.

In this Letter, a new method for the generation of OVs using optical fibers is presented. The OVs are generated uniquely by an optical fiber that has a SPP carved on the tip. This simple system aims to replace large and costly setups. Simultaneously, this Letter is motivated by the need to have beam tailoring tools for further use in the area of optical manipulation with fibers. This Letter is organized as follows: first, simulations modeling light propagation through a fiber with the SPP are presented, 
followed by the description of the fabrication methodology, the plate characterization, and subsequent study of the output field profile; finally, some closing remarks are discussed.

A SPP is a structure composed by helical phase steps, resulting in a thickness increasing proportionally to the azimuthal angle $\phi$ [Fig. 1(a)]. The plate has a maximum step height $s$, given by $l \lambda /\left(n_{\mathrm{SPP}}-n_{\text {medium }}\right)$, where $l$ represents the topological charge of the produced vortex, whereas $n_{\mathrm{SPP}}$ and $n_{\text {medium }}$ are the refractive index of the plate and the medium, respectively. With a SPP, Gaussian beams $(l=0)$ can be shaped into higher-order beams $(l \neq 0)$, and already existing LG modes can be reshaped into other orders.

In this particular case, the SPP is projected to produce a $l=1$ charged vortex (at $980 \mathrm{~nm}, n_{\mathrm{SPP}}=1.457$, in air), thus having a maximum step size of $2 \mu \mathrm{m}$. With the purpose of investigating the performance of the SPP, a solver of the Maxwell's equations, based on the finite difference time domain (FDTD) method (Lumerical), was used. The parameters used to model the fiber match the experimental data given by the fiber supplier (Corning HI $980 \mathrm{~nm}$ ). The SPP is $3.5 \mu \mathrm{m}$ wide (matching the core diameter), with depth increasing azimuthally in phase steps of $0.483 \mathrm{rad}$, corresponding to 13 levels, going from zero to a maximum step height of $2 \mu \mathrm{m}$ at $2 \pi$. Figure 2 shows the output electric field intensity in the $\mathrm{ZY}, \mathrm{YX}$, and $\mathrm{ZX}$ planes, where $\mathrm{X}$ is the propagation axis. In Figs. 2(a)-2(d), consecutive cross sections (ZY) along the propagation distance of the output electric field intensity are presented. In the near field, Figs. 4(a)-4(c), the intensity output is characterized by a doughnut shape $(3.7 \mu \mathrm{m}$ of outer diameter), preserving the intensity null $(0.5 \mu \mathrm{m}$ diameter $)$ at the center. Regarding the far-field region shown in Fig. 4(d), the profile corresponds to a spiral-shaped intensity distribution. The external rings visible in the ZY plane are likely due to diffraction in the boundaries of the plate. These features are confirmed by the YX and ZX cross sections, visible in Figs. 4(e) and 4(f), respectively. The areas marked with the dotted rectangles identify the zero intensity regions, characteristic of the phase singularities of OVs. The structure is well preserved over the first $2 \mu \mathrm{m}$; after this, the field starts twisting.

To fabricate the SPP, the tip of the fiber (Corning HI $980 \mathrm{~nm}$ ) is first cleaned and cleaved. Then, a 9.6\% hydrofluoric acid etchant gel is used to delimit the core of the fiber. Since the fiber core is etched more rapidly than the cladding, and this gel has a slow etching rate, after $15 \mathrm{~s}$, a nanoscale circular depression is visible on the surface of the fiber, matching the core location [23]. After this, the fibers are rinsed in both water and ethanol. To fabricate the SPP on the optical fibers, a dual beam-scanning electron microscope, also equipped with a focused ion beam (Quanta 3D, FEI Company), is used. The fibers are first coated with an organic conductor polymer, PEDOT: PSS (poly(3,4-ethylene dioxythio-phene):poly(stryrene sulfonate)), by dip coating to guarantee a good conductive surface and avoid charging effects during the milling and imaging processes [23]. The final goal of this process is to have a silica fiber with the structure milled on its surface, without any trace of the conductive film, avoiding back reflections. The PEDOT is water soluble; thus, after milling the surface, it is possible to remove it by dipping the fiber tip in water. During the milling, the conductivity properties of the PEDOT did not assure the ideal conditions for the fabrication of the phase plate, namely on the sharp discontinuity near the center of the pattern, due to the
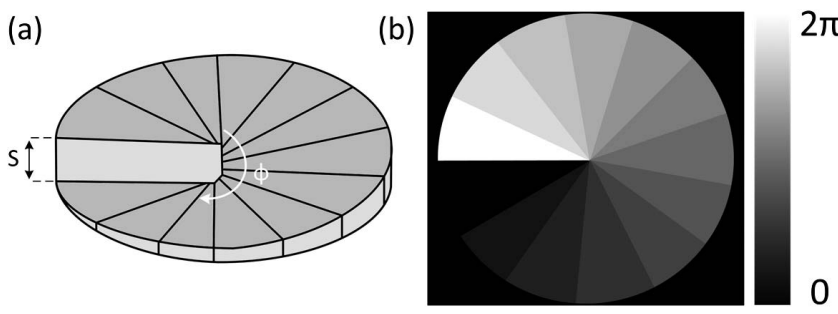

Fig. 1. (a) Sketch of a spiral phase plate used to generate vortex beams. (b) Phase distribution of the desired SPP.

ion beam induced charging and beam drift. Therefore, an extra film of gold-palladium, $5 \mathrm{~nm}$ thickness, was deposited onto the surface of the fiber, covering the PEDOT layer. Overall, this two-step process ensures good fabrication conditions and, by doing a lift-off process, the metallic film is removed. It is possible to mill complex structures using the FIB by uploading a bitmap file with the pattern characteristics. The pattern imprinted on the top of the fiber is shown in Fig. 1(b). The exposure dose is controlled by the dwell time, which is proportional to the bitmap pixel value. For a black pixel (level 0), the beam is blanked. For a white pixel (level 255), the dwell time is set to $1 \mu$ s. The dwell time for the pixel values in between is linearly interpolated. The ion beam current was set to $0.1 \mathrm{nA}$, which corresponded to a beam spot size of $24 \mathrm{~nm}$, allowing an optimal trade-off between patterning resolution and patterning duration. The milling process lasted for $29 \mathrm{~min}$, resulting in the structure depicted in Fig. 3(a). In Fig. 3(b), one can see a top view of the SPP and, in Fig. 3(c), a side view is visible, showing the features of the plate. The plate, with a diameter of $8 \mu \mathrm{m}$, is centered on the fiber core [Fig. 3(b)], and the maximum step height(s) achieved during the milling process is $1.6 \mu \mathrm{m}$ [Fig. 3(c)]. The diameter of the plate is larger than the core of the fiber $(3.5 \mu \mathrm{m})$ to avoid diffractive contributions from the plate edges. In Fig. 3(b), one can see that the center of the plate is slightly eroded. This results from the multiple scans of the ion beam over the entire structure during the milling process.
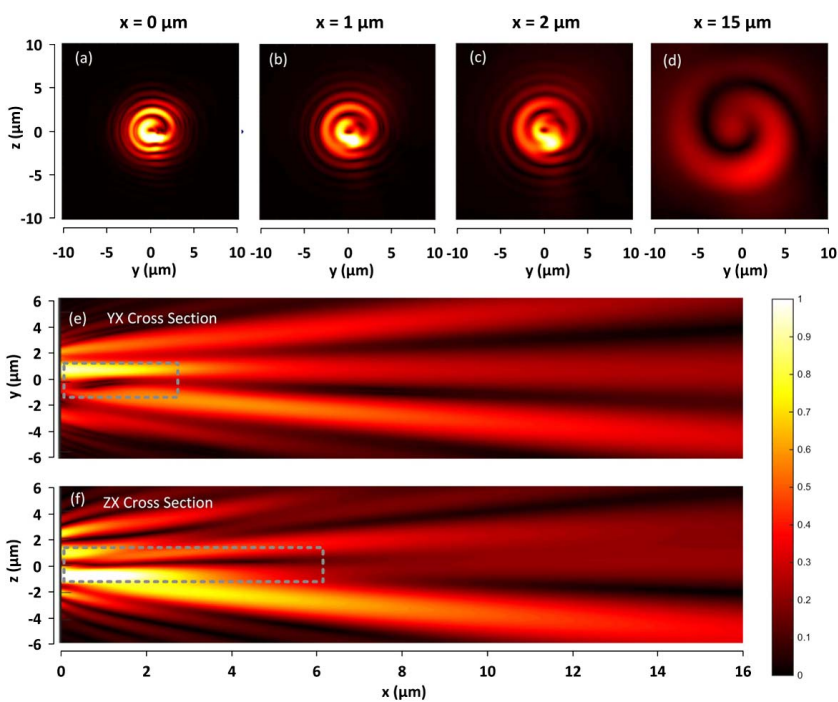

Fig. 2. $3 \mathrm{D}$ simulations obtained by Lumerical FDTD solver. (a)-(d) Snapshots of the electric intensity profile of the optical fiber tip output. Tangential and sagittal views $(\mathrm{X}$ is the propagation direction) of the output profile: (e) YX and (f) ZX. 

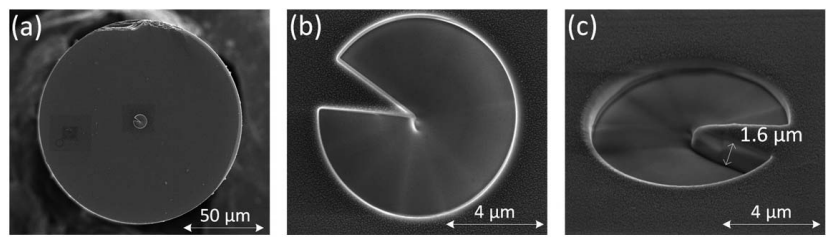

Fig. 3. (a) SEM image of the optical fiber with the SPP. (b) Zoom of the SPP (top view). (c) Zoom of the SPP (side view).

Sharper edges can be obtained by protecting the glass region of interest with an ion beam induced platinum deposition, which requires a strong acid mixture (aqua regia) for its removal, after the milling is complete. For this reason, it has been avoided.

To analyze the output beam profile, the fiber tip was spliced into a pigtailed diode laser (at $980 \mathrm{~nm}$ ), and the field was projected, using a $60 \times$ objective, into an infra-red camera. After this, the beam was scanned axially using a micrometer positioner, and snapshots of the output field were taken at each $1 \mu \mathrm{m}$, along $14 \mu \mathrm{m}$. The resulting output profile is visible in Fig. 4(a). The mode field diameter is $4.2 \mu \mathrm{m}$, corresponding to the radius of the SPP, failing to illuminate the borders of the plate. Work developed by Brasselet et al. also demonstrated that keeping the illuminating beam waist diameter smaller than the SPP increased the quality of the output vortex [15]. Overall, the field has a doughnut-shaped profile with asymmetric intensity distribution, and is slightly focused. Doing a reconstruction of the intensity profile, through the interpolation of the images using ImageJ software, allows a better perception of the OV features. In Figs. 4(b) and 4(c), the cross sections YX and ZX are presented. Analyzing the YX and ZX cuts, the central zero intensity, characteristic of OVs, is evident along the $14 \mu \mathrm{m}$. From 0 to $4 \mu \mathrm{m}$, the annular intensity outline is highly asymmetric, showing almost a distorted profile. In the range of 4-6 $\mu \mathrm{m}$ the features of the doughnut are more regular, and the side dimensions are reduced, suggesting a focusing area. The outer diameter of the annular field is approximately $6 \mu \mathrm{m}$, and the inner diameter corresponds to $1 \mu \mathrm{m}$. Finally, from 6 to $14 \mu \mathrm{m}$, the field diverges, and the annular profile vanishes.
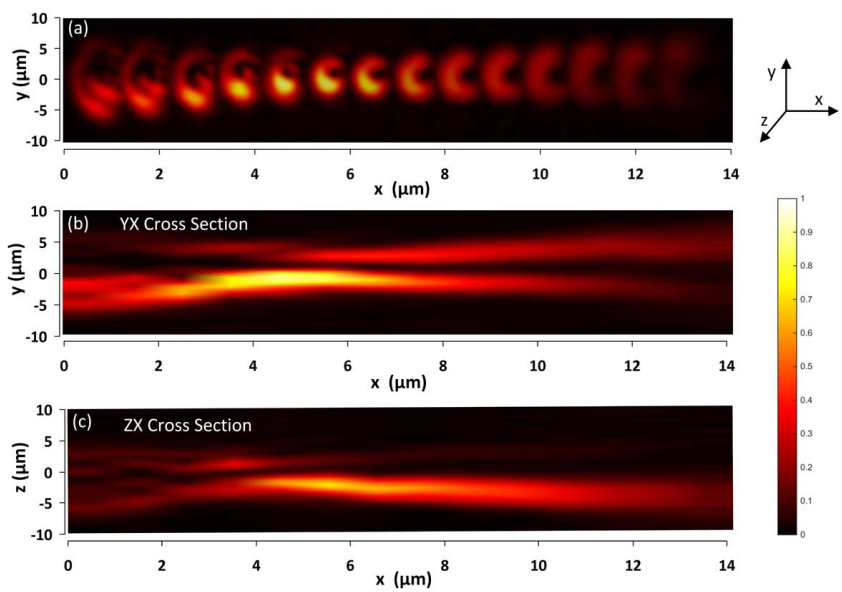

Fig. 4. (a) Snapshots of the experimental optical intensity profile of the optical fiber tip output. Orthogonal views of the output profile obtained through an interpolation of the field: (b) YX and (c) ZX. $\mathrm{X}$ is the main direction of light propagation.
This is more significant in the ZX plane and can be identified in Fig. 4(c).

On the basis of the results presented above, it is clear that the output optical intensity profile has some constraints regarding its symmetry. There are some possible causes, most probably associated with the fabrication process. First, a closer look into Fig. 3(b) shows that the SPP is misaligned with the fiber core by a $300 \mathrm{~nm}$ shift to the left. Therefore, when the fundamental Gaussian mode faces the spiral phase plate, the intensity peak will not be centered with the phase singularity of the plate, and an uneven distribution of the optical profile can arise. Second, the maximum step depth does not correspond exactly to a $2 \pi$ phase shift, due to unavoidable fabrication errors, and the nonuniform discretization of the number of levels on the phase plate introduces some degree of phase error [24]. Likewise, the deformation in the center of the plate, caused by the multiple scans of the FIB over the fiber surface, can cause a flawed doughnut shape.

Apart from these traits, the SPP also imprints a focusing outline on the electric field intensity. This effect is due to a curvature of the SPP caused during the milling process. In the course of the scanning of the ion beam over the fiber tip, there is always a higher milling near the edges. Comparing the computational and experimental results, there is an agreement regarding the extent of vortex and the general profile, where the doughnut shape is observable in both cases. Nonetheless, the focus effect was achieved only experimentally for the reasons previously explained. Particularly, the simulations presented here model an ideal spiral $2 \pi$ phase plate, with gradual phase step increase, centered in the fiber tip. In contrast, experimentally, the resulting SPP had some limitations, as indicated above, which were not accounted for in the model and can explain the discrepancies observed.

While the intensity profile of the output optical beam is studied using a magnification system and a CCD IR camera to acquire the images, the phase profiles are assessed using an interferometer. The literature on beams carrying orbital angular momentum abounds with examples of such measurement systems [4]. In this case, the interference of the beam carrying the $\mathrm{OV}$ and a reference beam will result in a characteristic pattern.

The setup used to test the SPP is presented in Fig. 5(a). The $980 \mathrm{~nm}$ diode laser is first connected to an optical fiber coupler $(50 / 50$ at $980 \mathrm{~nm})$. After this, one of the fibers is cleaved and is used as the reference beam, while the other is spliced at the SPP tip. The fibers are then placed on two micromanipulators and two $20 \times$ objectives are used to collimate the output beams. Finally, a beam splitter is used to recombine the beams, and the interference pattern is generated. The result is projected onto a CCD camera. Please note that the lengths of the interferometer arms are adjusted to match. Figures 5(b) and 5(c) show the reference (Gaussian profile) and the SPP output beam (doughnut shape), respectively. The coaxial interference of these two beams originates the pattern of Fig. 5(d). In this case, the interference fringes nucleate in a spiral profile, where the number of fringes defines the charge of the vortex. In this case, there is only one fringe, indicating that the beam should carry a vortex of charge 1 . This can be confirmed by the off-axis interference of the two beams that, according to the literature, must create a pattern with a discontinuity caused by the vortex, visible through the presence of degenerated/extra fringes, which are commonly called "forklets" [25]. Figure 5(e) shows the 
(a)
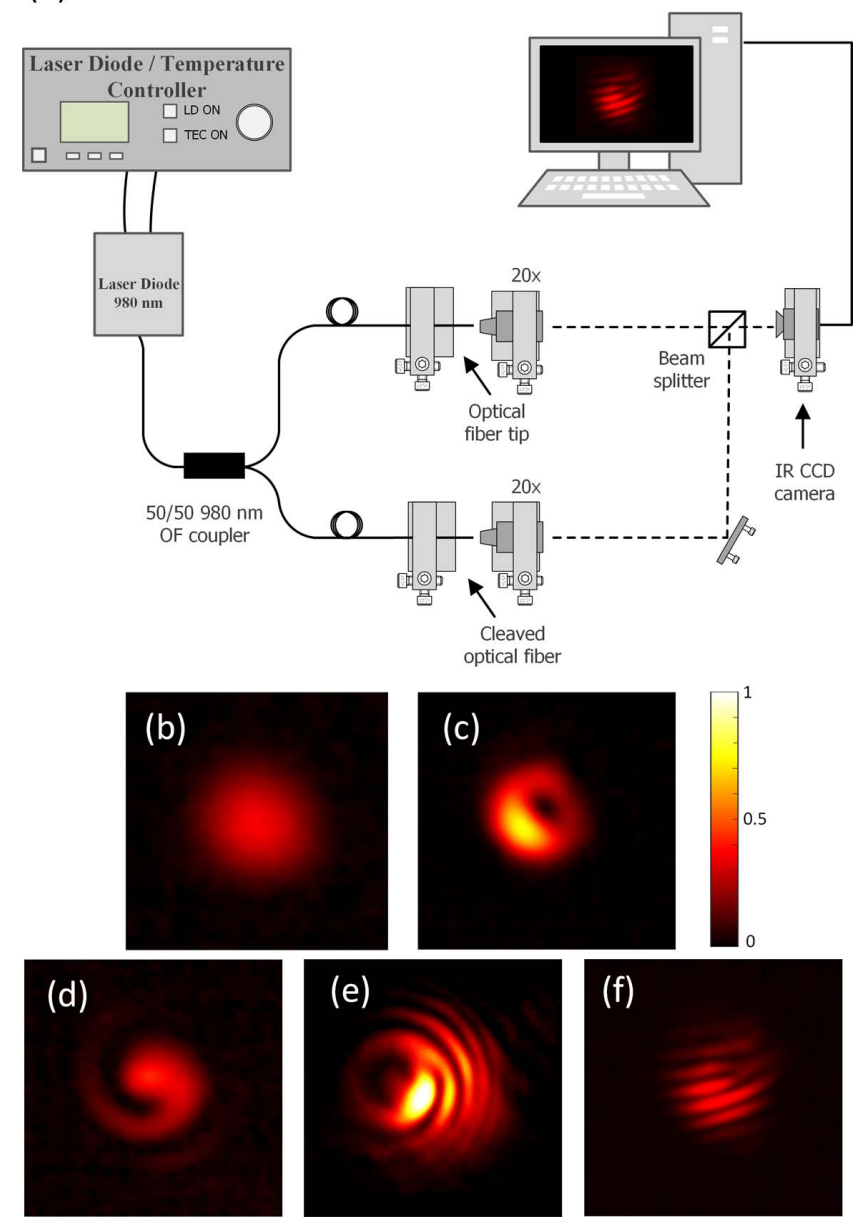

Fig. 5. (a) Interferometer scheme used to study the vortex charge. (b) Reference beam, (c) doughnut-shaped beam, (d) axial interference, (e) transition from axial to off-axis interference, and (f) off-axis interference.

transition between the coaxial and off-axis interference. Finally, after increasing the angle between the two beams, the forklet structure is visible, confirming the presence of the phase singularity that constitutes the OV.

The obtained results validate that SPP fabricated on optical fiber tips are able to transform Gaussian beams into a combination of Gaussian and Laguerre-Gaussian beams. From the analysis of the electric field intensity and the interferometric measurements, it can be said that the output is a $\mathrm{LG}_{01}$. Therefore, the generation of higher-order modes should be possible by increasing the height of the phase steps of the SPP, the number of steps and also by improving the fabrication conditions to avoid the misalignment and the nonuniform phase steps height. The number of phase steps is an important parameter with high impact on the mode conversion efficiency, as demonstrated by Scipioni et al. [24], where they found that 16 or 32 are values that highly increase the purity of the modes for their particular case. A more detailed study, with calculation and measurements of mode purity, is essential to assess the full potential of the devices and will be the subject of future work.

In a nutshell, this work describes a straightforward method for the generation of LG modes based on optical fibers with spiral phase plates. The fabrication method is based on the micromachining of optical fiber tips with the focused ion beam. In this particular case, the optical fiber tip was projected to tailor a Gaussian beam into the $\mathrm{LG}_{01}$ mode, which was demonstrated experimentally. The experimental results were preceded by computational simulations, indicating the potential of the expected outcomes.

Funding. North Portugal Regional Operational Programme (NORTE-01-0145-FEDER-000016); Portuguese Science and Technology Foundation (SFRH/BD/87542/ 2012); Masdar Institute of Science and Technology.

\section{REFERENCES}

1. J. H. Poynting, Proc. R. Soc. Lond. 82, 560 (1909).

2. R. A. Beth, Phys. Rev. 50, 115 (1936).

3. L. Allen, M. W. Beijersbergen, R. J. C. Spreeuw, and J. P. Woerdman, Phys. Rev. A 45, 8185 (1992).

4. A. M. Yao and M. J. Padgett, Adv. Opt. Photon. 3, 161 (2011).

5. M. R. Dennis, K. O'Holleran, and M. J. Padgett, Prog. Opt. 53, 293 (2009).

6. N. B. Simpson, K. Dholakia, L. Allen, and M. J. Padgett, Opt. Lett. 22 52 (1997)

7. A. V. Arzola, P. Jákl, L. Chvátal, and P. Zemánek, Opt. Express 22 16207 (2014).

8. X. Liu, X. Liu, L.-C. Kwek, and C. H. Oh, Front. Phys. China 3, 113 (2008).

9. D. Andrews, Structured Light and Its Applications (Elsevier, 2008).

10. S. Li and Z. Wang, Appl. Phys. Lett. 103, 141110 (2013).

11. N. Londoño, E. Rueda, J. A. Gómez, and A. Lencina, Appl. Opt. 54, 796 (2015).

12. R. K. Tyson, M. Scipioni, and J. Viegas, Appl. Opt. 47, 6300 (2008).

13. J. Courtial and M. J. Padgett, Opt. Commun. 159, 13 (1999).

14. K. Sueda, G. Miyaji, N. Miyanaga, and M. Nakatsuka, Opt. Express 12, 3548 (2004)

15. E. Brasselet, M. Malinauskas, A. Žukauskas, and S. Juodkazis, Appl. Phys. Lett. 97, 211108 (2010).

16. S. Ramachandran and P. Kristensen, Nanophotonics 2, 455 (2013).

17. C. N. Alexeyev, A. N. Alexeyev, B. P. Lapin, G. Milione, and M. A. Yavorsky, Phys. Rev. A 88, 063814 (2013).

18. Y. V. Kartashov, V. A. Vysloukh, and L. Torner, Opt. Lett. 38, 3414 (2013).

19. C. N. Alexeyev and M. A. Yavorsky, Phys. Rev. A 78, 043828 (2008).

20. R. S. Rodrigues Ribeiro, O. Soppera, A. G. Oliva, A. Guerreiro, and P. A. S. Jorge, J. Lightwave Technol. 33, 3394 (2015).

21. S. K. Mohanty, K. S. Mohanty, and M. W. Berns, Opt. Lett. 33, 2155 (2008).

22. S. Cabrini, C. Liberale, D. Cojoc, A. Carpentiero, M. Prasciolu, S. Mora, V. Degiorgio, F. De Angelis, and E. Di Fabrizio, Microelectron. Eng. 83, 804 (2006).

23. R. Janeiro, R. Flores, A. R. Ribeiro, P. Jorge, and J. Viegas, SPIE OPTO, G. von Freymann, W. V. Schoenfeld, R. C. Rumpf, and H. Helvajian, eds. (SPIE, 2015), p. 93740E.

24. M. Scipioni, R. K. Tyson, and J. Viegas, Appl. Opt. 47, 5098 (2008).

25. G. V. Bogatiryova and M. S. Soskin, Semicond. Phys. Quantum Electron. Optoelectron. 6, 254 (2003). 